\title{
Characterization of antioxidant olive oil biophenols by spectroscopic methods
}

\author{
Fátima Paiva-Martins, ${ }^{a}$ Vera Rodrigues, ${ }^{a}, b, c$ Rita Calheiros ${ }^{c}$ \\ and Maria PM Marques ${ }^{\mathrm{c*}}$
}

\begin{abstract}
BACKGROUND: Olive oil contains numerous phenolic components with well-recognized health-beneficial activity. The major phenolic compounds present in olives and virgin olive oil - hydroxytyrosol, oleuropein and the oleuropein aglycones 3,4DHPEA-EA and 3,4-DHPEA-EDA - as well as some of their metabolites were studied in the present work, regarding their main structural preferences. Vibrational spectroscopy (Raman) coupled to theoretical methods were used, aiming at fully characterizing the systems and therefore enabling their quick and reliable identification in food samples.

RESULTS: The Raman data, assisted by the theoretical simulations, allowed us to obtain the main geometrical and spectroscopic features of the olive oil constituents under study, which determine their known antioxidant and chemoprotective properties. In fact, it was verified that the spectra comprise distinctive bands for each compound, allowing their ready detection and differentiation.

CONCLUSION: This is the first reported study on the structural behaviour of olive oil phenolic compounds, and it established Raman spectroscopy as a rapid, non-destructive and reliable analytical technique for identifying these bioactive components in dietary extracts. It can surpass other analytical methods currently used, once it allows the concomitant identification of several olive oil components in a particular sample.

(c) 2010 Society of Chemical Industry
\end{abstract}

Keywords: olive oil; hydroxytyrosol; oleuropein; phenolic compounds; Raman spectroscopy; theoretical calculations

\section{INTRODUCTION}

Olive oil contains biophenolic components which were found to exert numerous beneficial biological effects, both in vitro and in vivo, ${ }^{1-4}$ including the prevention of severe pathologies such as cardiovascular distress and cancer, ${ }^{1,3}$ due to their high antioxidant capacity. ${ }^{2}$ These polyphenolic compounds comprise hydroxytyrosol (3,4-di-hydroxyphenyletanol, 3,4-DHPEA or HT) and its secoiridoid derivatives, namely oleuropein (OP) and the oleuropein aglycones 3,4-DHPEA-EA (elenolic acid linked to hydroxytyrosol) and 3,4-DHPEA-EDA (dialdehydic form of elenolic acid linked to hydroxytyrosol) (Figs 1 and 2).

The discovery of antioxidant activities (in vitro, ex vivo and in animal models) for the most important olive oil phenolic derivatives has stimulated intensive research on their presence in olive oils and on their bioavailability, metabolism and disposition in humans. The basis for these studies is that the debate on the potential benefits of olive oil phenolic compounds for human health require the support of clinical studies on their bioavailability. Initial investigations, performed both in animals and in humans, ${ }^{5}$ have shown that olive oil biophenols are mainly absorbed from the intestinal tract. Hydroxytyrosol rises early after ingestion, reaching a peak after ca. $1 \mathrm{~h}$ in plasma ${ }^{6}$ and up to $2 \mathrm{~h}$ in urine. ${ }^{7}$ Vissers and his collaborators have suggested that an important step in the metabolism of the olive oil phenolics oleuropein-glycoside and oleuropein-aglycones is their conversion into hydroxytyrosol. ${ }^{8}$ Following reports on HT metabolic disposition in humans, ${ }^{6-8}$ it was verified that two of the main competing pathways are $O$-glucuronide conjugation by uridine-5'-diphosphoglucuronyl transferases (UDPGTs), and $O$-methylation by catecholmethyltransferase (COMT), leading to the formation of homovanillyl alcohol (HVA). Furthermore, homovanillic acid and homovanillyl alcohol have been measured in human and animal studies, both in plasma and in urine, after olive oil ingestion. ${ }^{6-8}$ Therefore, the potential health benefits of olive oil phenolic derivatives may also be attributed to their conjugated metabolites or to the products of their enzymatic hydrolysis at the cellular level, which display a free phenolic moiety.

Despite the well-recognized activity of OVPs, very few studies are to be found in the literature on the effect of each particular phenolic component, and even fewer on the relationship between their activity and their structural characteristics and con-

\footnotetext{
* Correspondence to: Maria PM Marques, Department of Life Sciences, Faculty of Science and Technology, University of Coimbra, Ap. 3046, 3001-401 Coimbra, Portugal.E-mail:pmc@ci.uc.pt

a CIQ, Department of Chemistry and Biochemistry, Faculty of Sciences, University of Porto, Portugal

b Department of Pharmacy, College of Health Technology, Coimbra, Portugal

c Molecular Physical-Chemistry, Department of Life Sciences, University of Coimbra, Portugal
} 


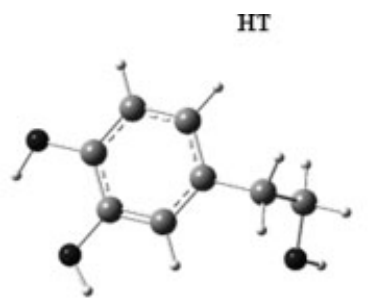

DOPAC
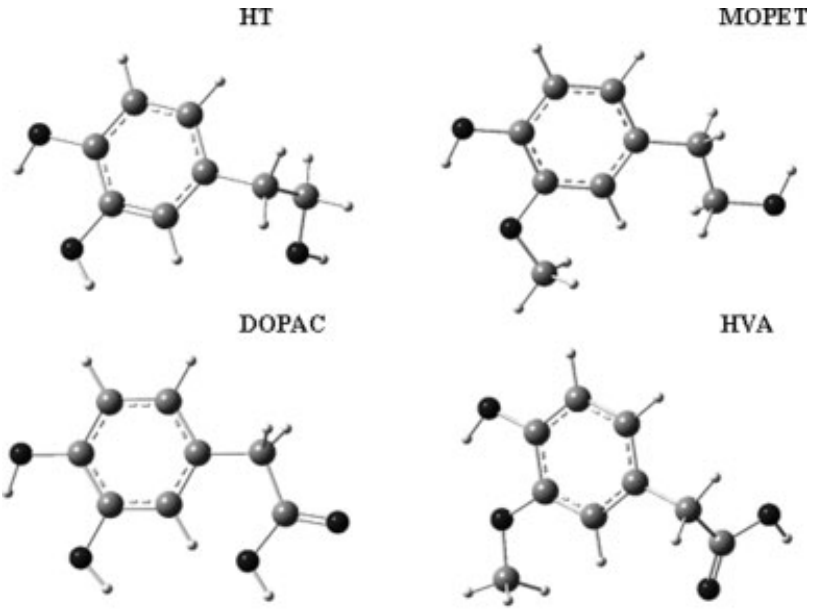

$\mathrm{C} \odot \mathrm{O}, \mathrm{H}$

Figure 1. Schematic representation of the most stable structures calculated for hydroxytyrosol (HT) and its metabolites MOPET, HVA and DOPAC.

formational behaviour. Recent in vitro studies ${ }^{9-11}$ have shown a significant protection towards the HL-60 cell line, erythrocytes and mononuclear blood cells by 3,4-DHPEA-EDA. This hydroxytyrosol ester has shown the best activity in these biological systems when compared to either hydroxytyrosol or oleuropein and the oleuropein aglycone 3,4-DHPEA-EA. These three secoiridoid molecules display a common 3,4-dihydroxyphenylethanol moiety, mainly responsible for their antiradical properties as well as for their ability to interact with both membranes and proteins, ${ }^{10}$ which seems to be mediated also by the elenolic acid group. Therefore, understanding the effect and mode of action of this kind of antioxidant molecules relies on the corresponding structure-activity relationships, which require a detailed charac- terization of the systems at the molecular level. It is therefore of the utmost importance to determine the conformational preferences of this kind of systems, both in the condensed phase and in solution, and to develop techniques which will allow a quick and reliable identification of each particular olive oil component in a biological sample (either a dietary extract or olive oil itself).

Raman spectroscopy has proven to be a highly reliable method for the determination of the composition profile of a sample, either solid or in solution (including aqueous solutions), ${ }^{12-16}$ owing to its non-invasiveness, high sensitivity and good reproducibility, coupled with the fact that it needs virtually no sample preparation. In fact, this technique yields unique fingerprint spectra, specific for each molecule, and can therefore be applied to both pure compounds and mixtures.

The present work reports a structural analysis of the main olive oil polyphenolic constituents hydroxytyrosol (2-(3,4dihydroxyphenyl)ethanol, HT) and its metabolites homovanillyl alcohol (2-(4-hydroxy-3-methoxiphenyl)ethanol, MOPET), homovanillic acid (4-hydroxy-3-methoxiphenylethanoic acid, HVA) and 3,4-dihydroxyphenylatanoic acid (DOPAC) (Fig. 1), as well as of the secoiridoid derivatives oleuropein (OP) and the oleuropein aglycones 3,4-DHPEA-EA and 3,4-DHPEA-EDA (Fig. 2). The main conformational preferences of these systems were obtained by coupling Raman spectroscopic data to the results yielded by theoretical methods (at the quantum mechanical level).

\section{EXPERIMENTAL \\ Phenolic compounds}

Hydroxytyrosol was synthesized from 3,4-dihydroxyphenylacetic acid (Sigma-Aldrich Quimica-SA, Madrid, Spain) according to the procedure of Baraldi et al. ${ }^{17}$

Oleuropein was extracted from olive leaves following the method of Gariboldi et al. ${ }^{18}$ The aglycone 3,4-DHPEA-EA was
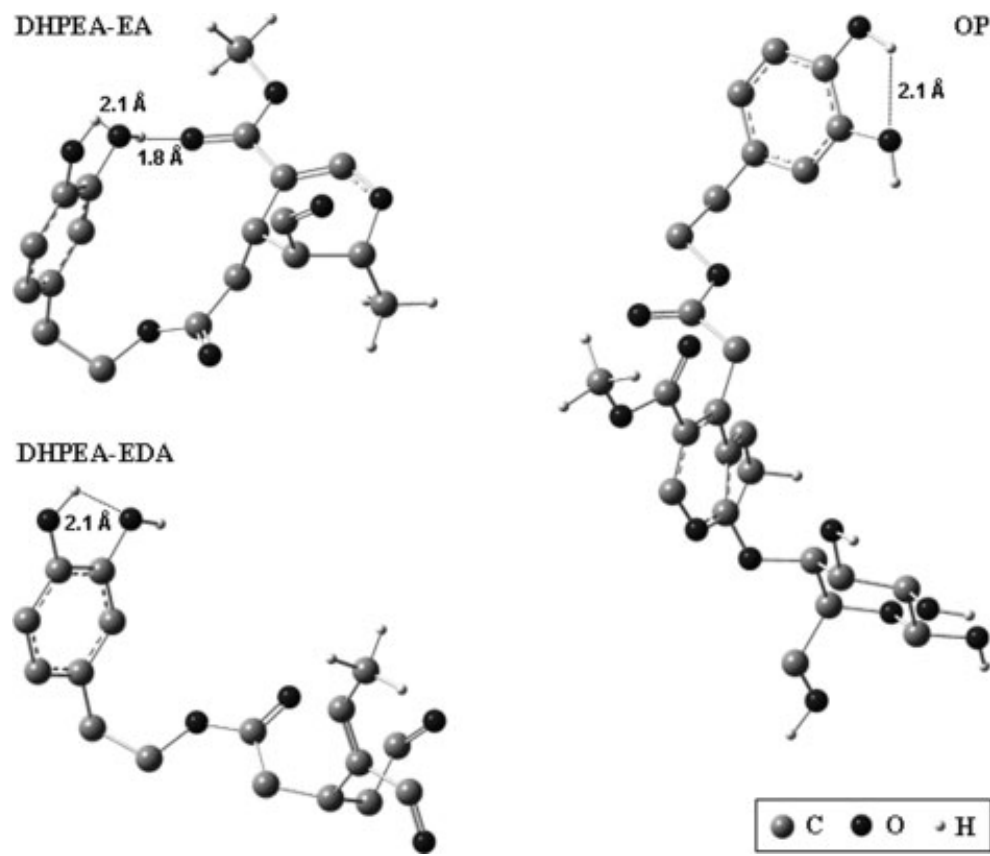

C $\bullet \circ, \mathrm{H}$

Figure 2. Schematic representation of the most stable geometries calculated for the olive oil secoiridoids OP, 3,4-DHPEA-EA and 3,4-DHPEA-EDA. (For clarity, most hydrogen atoms are not shown). 
obtained from oleuropein by enzymatic reaction using $\beta$ glycosidase (Fluka, Buchs, Switzerland), according to the method reported by Limirioli et al. ${ }^{19}$ 3,4-DHPEA-EDA was extracted from olive leaves, following the process of Paiva-Martins and Gordon. ${ }^{20}$

\section{Theoretical calculations}

Theoretical (quantum mechanical) calculations were performed in order to obtain a reliable structural model of the polyphenolic systems studied and simulate the corresponding vibrational spectra. The Gaussian $03 \mathrm{~W}$ program ${ }^{21}$ was used, within the Density Functional Theory (DFT) approach, which accounts for the electron correlation effects that are particularly important in this kind of conjugated systems. The hybrid method denoted by B3LYP, including a mixture of HF and DFT exchange terms and the gradient-corrected correlation functional of Lee et al., ${ }^{22,23}$ as proposed and parametrized by Becke, ${ }^{24,25}$ was applied, along with the double-zeta split valence basis set $6-31 \mathrm{G} * * 26,27$

Molecular geometries were fully optimized by the Berny algorithm, using redundant internal coordinates: ${ }^{28}$ the bond lengths to within $c a .0 .1 \mathrm{pm}$ and the bond angles to within ca. $0.1^{\circ}$. The final root mean square (rms) gradients were always less than $3 \times 10^{-4}$ hartree bohr ${ }^{-1}$ or hartree radian ${ }^{-1}$. No geometrical constraints were imposed on the molecules under study. The relative energies and populations (Boltzman distribution at $298.15 \mathrm{~K}$ ) were calculated for all conformers, using the sum of the electronic and zero-point energies.

Calculation of the harmonic vibrational frequencies was carried out for all geometries, in order to check for minima in the potential energy surface and to obtain the theoretical vibrational pattern (both Raman and infrared) of the compounds. Wavenumbers above $400 \mathrm{~cm}^{-1}$ were scaled by a factor of $0.9614,{ }^{29}$ before comparing them with the experimental data, in order to correct for the anharmonicity of the normal modes of vibration.

\section{Raman spectroscopy}

The Raman spectra were obtained at room temperature, in a triple-monochromator Jobin Yvon T64000 Raman system (Paris, France) (focal distance $0.640 \mathrm{~m}$, aperture $\mathrm{f} / 7.5$ ) with holographic gratings of 1800 grooves $\mathrm{mm}^{-1}$. The premonochromator stage was used in the subtractive mode. The detection system was a liquid-nitrogen-cooled non-intensified $1024 \times 256$ pixel ( $\left.1^{\prime \prime}\right)$ Charge Coupled Device (CCD). The entrance slit was set to $200 \mu \mathrm{m}$, and the slit between the premonochromator and the spectrograph was opened to $12 \mathrm{~mm}$.

The excitation radiation was provided (ca. $100 \mathrm{~mW}$ at the sample position) by the $514.5 \mathrm{~nm}$ line of an $\mathrm{Ar}^{+}$laser (model Innova 300,
Coherent Inc., Santa Clara, CA, USA). A $90^{\circ}$ geometry between the incident radiation and the collecting system was employed. Samples were sealed in Kimax glass capillary tubes of $0.8 \mathrm{~mm}$ inner diameter. Under the above-mentioned conditions, the error in wavenumbers was estimated to be within $1 \mathrm{~cm}^{-1}$.

Raman spectra were collected for both the solid samples and for solutions (in both water and dimethyl sulfoxide).

\section{RESULTS AND DISCUSSION}

Figures 1 and 2 represent the most stable structures calculated for the olive oil polyphenolic components presently investigated. Hydroxytyrosol and its metabolites (MOPET, HVA and DOPAC) (Fig. 1) display ring substituents - either $\mathrm{OH}$ or $\mathrm{OCH}_{3}$-coplanar with the aromatic ring (establishing $(\mathrm{O}) \mathrm{H} \cdots \mathrm{O}$ intramolecular interactions, $\mathrm{d}_{\mathrm{O}-\mathrm{H}} \mathrm{ca} .2 .1 \AA$ ), while the saturated pendant arm containing the hydroxyl (in the HT and MOPET alcohols) or carboxylate moieties (in DOPAC and HVA) is tilted by $90-120^{\circ}$ relative to the ring, thus minimizing unfavourable steric repulsions within the molecule. Actually, it is interesting to note that the only conformational difference detected between HVA and its unsaturated analogue ferulic acid $(F A)^{30}$ is the non-planar orientation of the $-\mathrm{CH}_{2} \mathrm{COOH}$ group in the former as opposed to the complete planarity of the FA molecule (Fig. 3), due to the presence of the carbon chain double bond, which favours a planar geometry through conjugation with the aromatic ring. While for HT and DOPAC two conformations are expected to occur at room temperature (with $80: 20 \%$ and $76: 24 \%$ relative populations, respectively; Table 1), only one geometry will be present for the MOPET and HVA metabolites. Moreover, the two RT-populated DOPAC geometries display quite distinct polarities, DOPAC2's dipole moment being approximately twice that of DOPAC1 (as opposed to HT; Table 1).

The larger secoiridoid molecules OP, 3,4-DHPEA-EA and 3,4DHPEA-EDA (Fig. 2) evidence a clear effect of particular geometrical parameters on their overall stability, namely: (i) the formation of intramolecular hydrogen-type bonds (e.g., (O) $\mathrm{H} \cdots \mathrm{O}$ and $(\mathrm{C}=) \mathrm{O} \cdots \mathrm{H}$; Fig. 2); (ii) the relative position of the rings (in 3,4DHPEA-EA and OP). These are highly tilted systems, the only common feature among them being the relative orientation of the phenolic hydroxyl groups, always coplanar with the aromatic ring (due to the possibility of occurrence of an $(\mathrm{O}) \mathrm{H} \cdots$ O intramolecular close contact).

The conformational behaviour of the compounds under study conforms to the expected preferences of this type of $\pi$-conjugated systems, the main determinant factors being the electronic effects

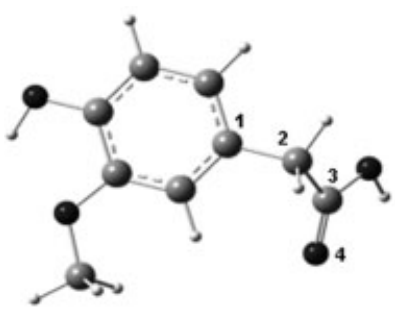

HVA

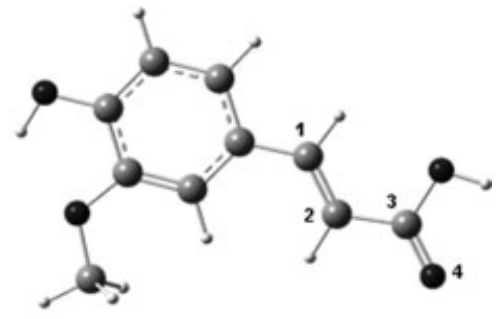

FA

$\mathrm{C} \odot \mathrm{O}, \mathrm{H}$

Figure 3. Schematic representation of the most stable structures obtained for $\mathrm{HVA}$ and ferulic acid $(\mathrm{FA}) .\left(\mathrm{C}_{1} \mathrm{C}_{2} \mathrm{C}_{3} \mathrm{O}_{4}\right.$ dihedral equal to $75.8^{\circ}$ and $0^{\circ}$ for HVA and FA, respectively). 
Table 1. Relative conformational energies, relative populations and total dipole moments ( $\mu$, in Debyes) for the most stable geometries calculated for HT, MOPET, HVA and DOPAC

\begin{tabular}{|lccc|} 
Conformer & $\Delta E\left(\mathrm{~kJ} \mathrm{~mol}^{-1}\right)$ & Population $(\%)$ & $\mu(\mathrm{D})$ \\
\hline HT1 & 0 & 80.4 & 3.0 \\
HT2 & 3.5 & 19.6 & 3.6 \\
MOPET1 & 0.0 & 99 & 1.5 \\
MOPET2 & 11.4 & 1 & 3.9 \\
MOPET3 & 23.7 & 0 & 3.6 \\
MOPET4 & 25.1 & 0 & 2.0 \\
HVA1 & 0 & 100 & 1.4 \\
HVA2 & 26.7 & 0 & 3.4 \\
HVA3 & 40.2 & 0 & 6.4 \\
DOPAC1 & 0 & 76.2 & 3.5 \\
DOPAC2 & 2.9 & 23.7 & 1.8 \\
DOPAC3 & 17.2 & 0.1 & 2.1 \\
\hline
\end{tabular}

and the possibility of occurrence of intramolecular hydrogen bonds. In fact, an extra stabilization was found to be achieved through medium-strength intramolecular $(\mathrm{C}) \mathrm{H} \cdots \mathrm{O}$ and $(\mathrm{H}) \mathrm{O} \cdots \mathrm{H}$ close contacts. The molecules are thus folded to a certain extent, as a consequence of the equilibrium between the attractive $\mathrm{O} \cdots \mathrm{H}$ and the repulsive $\mathrm{O} \cdots \mathrm{O}$ and $\mathrm{H} \cdots \mathrm{H}$ interactions.

Vibrational (Raman) data were obtained for each of the polyphenolic systems investigated, in order to assess their characteristic vibrational modes. Figure 4 comprises the Raman spectra of hydroxytyrosol and its metabolites MOPET, HVA and DOPAC, in the solid state, along with the simulated spectrum of HT. The Raman experimental pattern obtained for oleuropein, both in the condensed state and in aqueous solution, is represented in Fig. 5. The tentative assignment of the major vibrational bands detected for these compounds, which allow their quick and unequivocal identification, is summarized in Tables 2 and 3.

Some common Raman signals are observed for all the compounds studied, namely the main $v_{\mathrm{C}}=\mathrm{c}$ ring modes around 1670,1620 and $780 \mathrm{~cm}^{-1}$, and two distinct phenolic $\mathrm{OH}$ stretching bands at ca. $3650 \mathrm{~cm}^{-1}$ and ca. $3065 \mathrm{~cm}^{-1}$, the latter being ascribed to the hydroxyl group involved in intramolecular $\mathrm{H}$ bonds (responsible for the large shift to low frequencies). For hydroxytyrosol and its metabolites, the signal around $780 \mathrm{~cm}^{-1}$ also contains a contribution from the ring $\mathrm{OH}$ bending (Table 2). For systems containing carboxylate moieties, typical $\mathrm{OH}$ and $\mathrm{C}=\mathrm{O}$ stretching features are clearly detected at about $3550 \mathrm{~cm}^{-1}$ and $1650-1700 \mathrm{~cm}^{-1}$, respectively.

The Raman technique yields a spectral fingerprint for each of the olive oil constituents presently analysed (Tables 2 and 3). In fact, while HVA and MOPET yield distinct vibrational modes for the methoxyl group, at 1000 and $225 \mathrm{~cm}^{-1}$, corresponding to the $\mathrm{C}-\mathrm{O}\left(\mathrm{CH}_{3}\right)$ stretching and methyl torsion, respectively, only for HVA and DOPAC is the $\mathrm{C}=0$ stretching detected, at $c a .1650 \mathrm{~cm}^{-1}$ (Fig. 4). Moreover, the alcohol group in HT and MOPET gives rise to a characteristic $\nu_{\mathrm{OH}}$ mode at ca. $3700-3650 \mathrm{~cm}^{-1}$.

The major vibrational features characteristic of each molecule investigated were also detected in the aqueous solution spectra (Figs 5 and 6). Furthermore, a reasonably good accordance was verified between the experimental and the simulated data (Figs 4 and 5), which allows an accurate assignment of the experimental vibrational features by comparison with the simulated Raman pattern. Therefore, coupling the theoretical results to the spectroscopic data presently obtained for these polyphenolic systems leads to a thorough elucidation of their detailed geometrical preferences, which are directly related to their antioxidant properties and overall biological activity (e.g.,

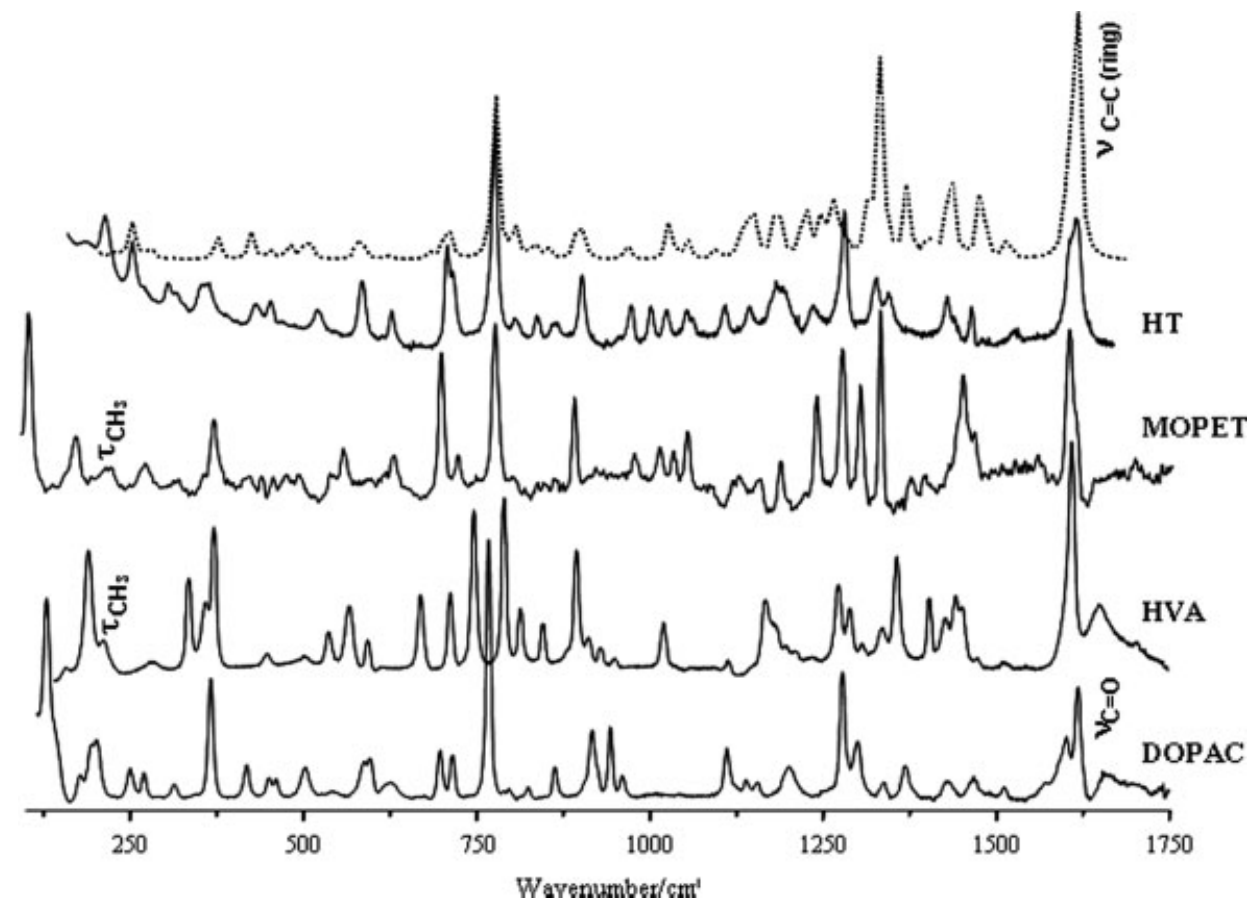

Figure 4. Experimental Raman spectra $\left(100-1750 \mathrm{~cm}^{-1}\right)$ for hydroxytyrosol $(\mathrm{HT})$ and its metabolites MOPET, HVA and DOPAC. (The dotted line represents the calculated spectrum for $\mathrm{HT}$ ). 


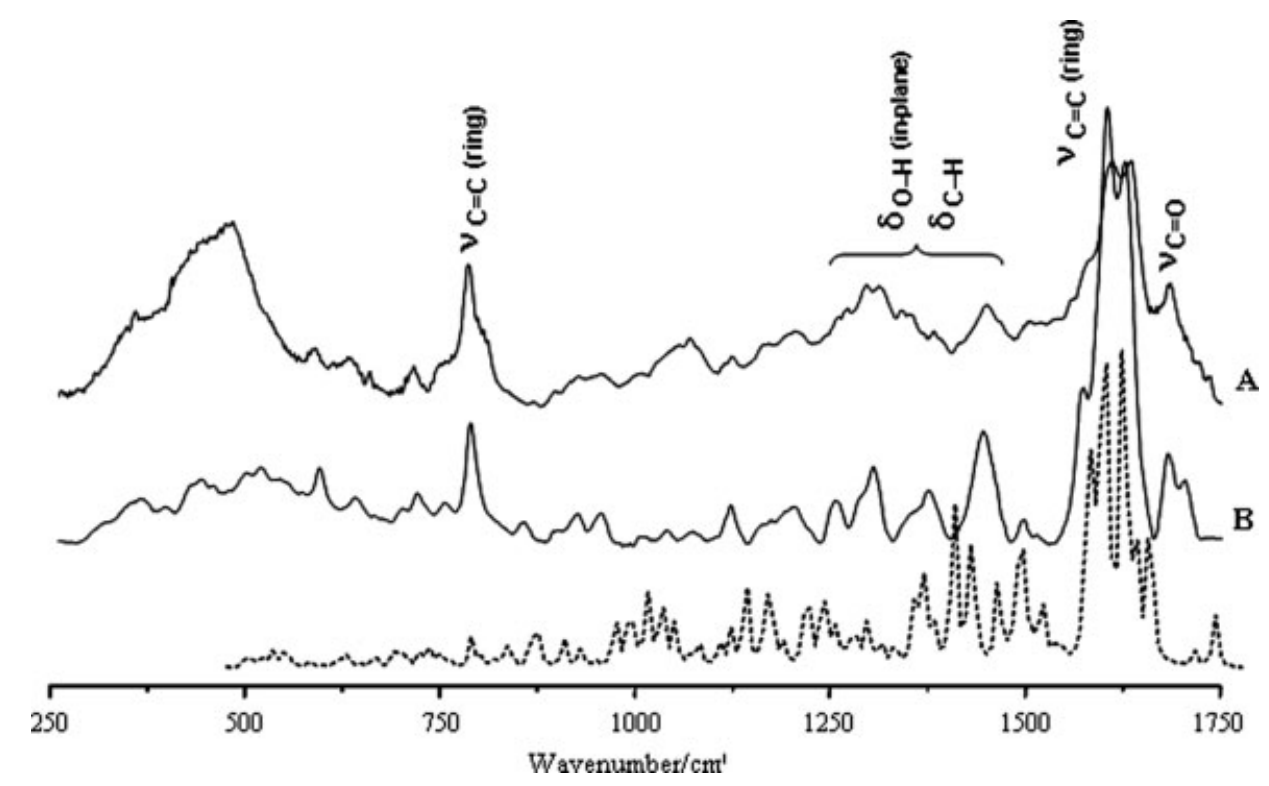

Figure 5. Experimental Raman spectra $\left(250-1750 \mathrm{~cm}^{-1}\right)$ for OP: A, $0.2 \mathrm{~mol} . \mathrm{dm}^{-3}$ aqueous solution; $\mathrm{B}$, condensed phase. (The dotted line represents the calculated spectrum).

\begin{tabular}{|c|c|c|c|c|}
\hline HT & MOPET & HVA & DOPAC & Tentative assignment \\
\hline 3700 & 3650 & & & $v(\mathrm{OH})_{\text {alcohol }}$ \\
\hline 3500 & 3551 & 3503 & 3551 & $v(\mathrm{OH})_{\text {ring }}$ \\
\hline \multirow[t]{2}{*}{3100} & 3071 & 3064 & 3069 & $v(\mathrm{OH})_{\text {ring }}(\mathrm{H}$-bonded $)$ \\
\hline & & 1651 & 1655 & $v(\mathrm{C}=\mathrm{O})$ \\
\hline 1613 & 1603 & 1613 & 1619 & $v(\mathrm{C}=\mathrm{C})_{\text {ring }}$ \\
\hline \multirow[t]{2}{*}{1329} & 1330 & 1362 & & $\delta(\mathrm{C}-\mathrm{H})$ \\
\hline & 980 & 1000 & & $v\left(\mathrm{C}-\mathrm{O}\left(\mathrm{CH}_{3}\right)\right)$ \\
\hline 781 & 780 & 794 & 768 & $v(\mathrm{C}=\mathrm{C})_{\text {ring }}+\delta(\mathrm{O}-\mathrm{H})_{\text {ring }}$ \\
\hline 712 & 702 & 753 & 717 & $\Gamma(\mathrm{CCC})+\Gamma(\mathrm{OCC})$ \\
\hline \multirow[t]{2}{*}{363} & 374 & 381 & 368 & $\Gamma(\mathrm{CCC})+\Gamma(\mathrm{OCC})$ \\
\hline & 225 & 220 & & $\tau\left(\mathrm{CH}_{3}\right)$ \\
\hline
\end{tabular}

the way they interact with particular receptors and biological targets).

To the best of the authors' knowledge, this is the first report of a comparative analysis of the structural characteristics of the main olive oil polyphenolic constituents. Actually, this study corroborates Raman spectroscopy as a method of choice for the detection and identification of this kind of phytochemicals in plant extracts or food products.

\section{CONCLUSIONS}

Raman spectroscopy combined with DFT calculations were applied to the characterization and analysis of the main conformational preferences of the olive oil constituents hydroxytyrosol and its metabolites, as well as of the secoiridoids OP, 3,4-DHPEA-EDA and 3,4-DHPEA-EA. With regard to the recognized structural dependence of the biological activity of these phenolic derivatives, namely as chemopreventive agents against oxidative-induced processes (e.g., neurodegenerative disorders and carcinogenesis), the information yielded by this type of study will help to
Table 3. Experimental Raman wavenumbers $\left(\mathrm{cm}^{-1}\right)$ and tentative assignments for the main bands of OP, 3,4-DHPEA-EA and 3,4-DHPEAEDA

\begin{tabular}{|lccl|}
\hline OP & 3,4-DHPEA-EA & 3,4-DHPEA-EDA & Tentative assignment \\
\hline 3567 & 3550 & 3524 & $v(\mathrm{OH})_{\text {ring }}$ \\
2983 & 2986 & 2944 & $v(\mathrm{CH})$ \\
2940 & 2954 & 2924 & $v(\mathrm{CH})$ \\
1734 & 1765 & 1716 & $v(\mathrm{C}=\mathrm{O})$ \\
1712 & 1720 & 1704 & $v(\mathrm{C}=\mathrm{O})$ \\
& & 1690 & $v(\mathrm{C}=\mathrm{O})$ \\
1683 & 1675 & 1671 & $v(\mathrm{C}=\mathrm{C})_{\text {ring }}$ \\
765 & 780 & 778 & $v(\mathrm{C}=\mathrm{C})_{\text {ring }}$ \\
719 & 712 & & $\Gamma(\mathrm{CCC})+\Gamma(\mathrm{OCC})$ \\
250 & 250 & 245 & $\tau\left(\mathrm{CH}_{3}\right)$ \\
& 220 & & $\tau\left(\mathrm{CH}_{3}\right)$ \\
\hline
\end{tabular}

understand the mechanisms through which phenols act in living systems, and hopefully establish structure-activity relationships governing this cytoprotective role. This type of spectroscopic analysis of polyphenolic systems greatly benefits from the use of theoretical simulations (e.g., by quantum mechanical methods), which yield additional data supporting an accurate assignment of the experimental spectra, leading to reliable structural models of the systems.

The present work, which is the first reported study on the structural behaviour of olive oil biophenols, established Raman spectroscopy as a promising tool for the identification of such compounds, since it has proved to enable rapid and nondestructive measurements in very small amounts of sample (either pure or in solution) leading to the detection of spectral features characteristic of each particular compound. In fact, even chemically similar phenolic molecules could be easily distinguished by this technique. Therefore, the method has the additional advantage of enabling its extension to the metabolites of a certain compound (e.g., detection of hydroxytyrosol and its main metabolites). It can 


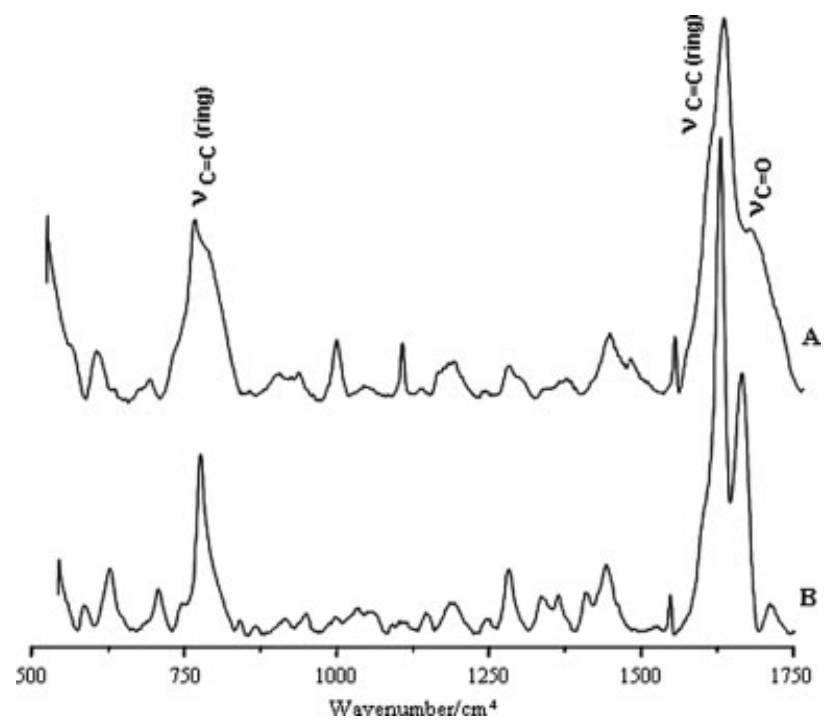

Figure 6. Experimental Raman spectra $\left(500-1750 \mathrm{~cm}^{-1}\right.$, saturated aqueous solution) for 3,4-DHPEA-EA (A) and 3,4-DHPEA-EDA (B).

also surpass other analytical methods currently used, once it allows the concomitant identification of several olive oil components in a particular olive oil sample.

\section{ACKNOWLEDGEMENTS}

The authors acknowledge financial support from the Portuguese Foundation for Science and Technology (PhD fellowship SFRH/BD/16520/2004).

\section{REFERENCES}

1 Owen RW, Haubner R, Würtele G, Hull WE, Spiegelhalder B and Bartsch $\mathrm{H}$, Olives and olive oil in cancer prevention. Eur J Cancer Prevent 13:319-326 (2004)

2 Covas Ml, Olive oil and the cardiovascular system. Pharmacol Res 55:175-186 (2007).

3 Pérez-Jiménez $F$, Ruano J, Perez-Martinez $\mathrm{P}$, Lopez-Segura $\mathrm{F}$ and Lopez-Miranda J, The influence of olive oil on human health: not a question of fat alone. Mol Nutr Food Res 51:1199-1208 (2007).

4 Obied HK, Prenzler PD, Konczakl, Rehman AU and Robards K, Chemistry and bioactivity of olive biophenols in some antioxidant and antiproliferative in vitro bioassays. Chem Res Toxico/ 22:227-234 (2009).

5 Tuck K and Hayball PJ, Major phenolic compounds in olive oil: metabolism and health effects. J Nutr Biochem 13:636-644 (2002).

6 Fitó M, De La Torre R, Farré-Albaladejo M, Khymenetz O, Marrugat J and Covas MI, Bioavailability and antioxidant effects of olive oil phenolic compounds in humans: a review. Ann Ist Super Sanita, 43:374-381 (2007)

7 Miro-Casas E, Covas MI, Farre M, Fito M, Ortuñ J, Weinbrenner T, et al, Hydroxytyrosol disposition in humans. Clin Chem 49:945-952 (2003).

8 Vissers MN, Zock PL, Roodenburg AJC, Leenen R and Katan MB, Olive oil phenols are absorbed in humans. J Nutr 132:409-417 (2002).

9 Fabiani R, Rosignoli P, De Bartolomeo A, Fuccelli R, Servili M, Montedoro GF, et al, Oxidative DNA damage is prevented by extracts of olive oil, hydroxytyrosol, and other olive phenolic compounds in human blood mononuclear cells and HL60 cells. J Nutr 138:1411-1416 (2008).
10 Paiva-Martins F, Fernandes J, Rocha S, Nascimento H, Vitorino R, Amado F, et al, Effects of olive oil polyphenols on erythrocyte oxidative damage. Mol Nutr Food Res 53:609-616 (2009).

11 Paiva-Martins F, Fernandes J, Rocha S, Borges F, Belo L and SantosSilva A, Powerful protective role of 3,4-dihydroxyphenylethanolelenolic acid dialdehyde against erythrocyte oxidative-induced hemolysis. J Agric Food Chem 58:135-140 (2010).

12 Bell SEJ, Burns DT, Dennis AC and Speers JS, Rapid analysis of ecstasy and related phenethylamines in seized tablets by Raman spectroscopy. Analyst 125:541-544 (2000).

13 Bell SEJ, Burns DT, Dennis AC, Matchett LJ and Speers JS, Composition profiling of seized ecstasy tablets by Raman spectroscopy. Analyst 125:1811-1815 (2000).

14 Sägmüller B, Schwarze B, Brehm G and Schneider S, Application of SERS spectroscopy to the identification of (3,4-methylenedioxy)amphetamine in forensic samples utilizing matrix stabilized silver halides. Analyst 126:2066-2071 (2001).

15 VankeirsbilckT, Vercauteren A, Baeyens W, van der Weken G, Verpoort F, Vergote G, et al, Applications of Raman spectroscopy in pharmaceutical analysis. Trends Anal Chem 21:869-877 (2002).

16 Milhazes N, Borges F, Calheiros R and Marques MPM, Identification of synthetic precursors of amphetamine-like drugs using Raman spectroscopy and $A b$ Initio calculations: $\beta$-methyl- $\beta$-nitrostyrene derivatives. Analyst 129:1106-1117 (2004).

17 Baraldi PG, Simoni D, Manfredini S and Menziani E, Preparation of 3,4 dihydroxy-1-benzeneethanol: a reinvestigation. Liebigs Ann Chem 684-686 (1983)

18 Gariboldi P, Jommi G and Verotta L, Secoiridoids from Olea europaea. Phytochemistry 25:865-869 (1986)

19 Limiroli R, Consonni R, Ottolina G, Marsilo V, Bianchi G and Zetta L, ${ }^{1} \mathrm{H}$ NMR and ${ }^{13} C$ NMR characterization of new oleuropein aglycones. J Chem Soc Perkin Trans 1 1519-1523 (1995).

20 Paiva-Martins F and Gordon $\mathrm{MH}$, Isolation and characterization of the antioxidant component 3,4-dihydroxyphenylethyl 4-formyl-3formylmethyl-4-hexenoate from olive (Olea europaea). J Agric Food Chem 49:4214-4219 (2001).

21 Frisch MJ, Trucks GW, Schlegel HB, Scuseria GE, Robb MA, Cheeseman JR, et al, G. Gaussian 03, Revision B.04. Gaussian, Inc., Pittsburgh PA (2003).

22 Lee C, Yang W and Parr RG, Development of the Colle-Salvetti correlation-energy formula into a functional of the electron density. Phys Rev B 37:785-789 (1988).

23 Miehlich B, Savin A, Stoll H and Preuss $H$, Results obtained with the correlation energy density functionals of Becke and Lee, Yang and Parr. Chem Phys Lett 157:200-206 (1989).

24 Becke AJ, Density-functional exchange-energy approximation with correct asymptotic behavior. Phys Rev A 38:3098-3100 (1988).

25 Becke AJ, Density-functional thermochemistry. III. The role of exact exchange. J Chem Phys 98:5648-5652 (1993).

26 Hariharan PC and Pople JA, The influence of polarization functions on molecular orbital hydrogenation energies. Theor Chim Acta 28:213-222 (1973)

27 Petersson GA, Bennett A, Tensfeldt TG, Al-Laham MA, Shirley WA and Mantzaris J, A complete basis set model chemistry. I. The total energies of closed-shell atoms and hydrides of the first-row elements. J Chem Phys 89:2193-2218 (1998).

28 Peng C, Ayala PY, Schlegel HB and Frisch MJ, Using redundant internal coordinates to optimize equilibrium geometries and transition states. J Comp Chem 17:49-56 (1996).

29 Scott AP and Radom L, Harmonic vibrational frequencies: an evaluation of Hartree-Fock, Møller-Plesset, quadratic configuration interaction, density functional theory, and semiempirical scale factors. J Phys Chem 100:16502-16513 (1996).

30 Calheiros R, Borges F and Marques MPM, Conformational behaviour of biologically active ferulic acid derivatives. J Mol Struct (THEOCHEM) 913:146-156 (2009). 